\title{
An efficient surface integral equation method for the time-harmonic Maxwell equations
}

\author{
M. Ganesh ${ }^{1} \quad$ S. C. Hawkins ${ }^{2}$
}

(Received July 28, 2006; revised April 10, 2007)

\begin{abstract}
We present an efficient algorithm to compute high order approximations to the radar cross section (RCS) of a perfectly conducting three dimensional target by solving the time harmonic Maxwell equations. We demonstrate that the algorithm requires only a few tens of thousands of unknowns to compute the RCS of three dimensional targets at medium frequencies. These relatively small linear systems can be solved iteratively or using a direct solver. The possibility of using a direct solver is an advantage in computing the monostatic RCS, which involves solving hundreds of linear systems with one matrix but hundreds of right hand sides. We demonstrate that the monostatic RCS of medium electromagnetic sized conductors can be computed at over one thousand transmitter directions in a few hours of computer time.
\end{abstract}

See http://anziamj.austms.org.au/ojs/index.php/ANZIAMJ/article/view/60 for this article, (c) Austral. Mathematical Soc. 2007. Published April 26, 2007. ISSN 1446-8735 


\section{Contents}

1 Introduction

C18

2 Surface integral equation

C20

3 High order fully discrete Galerkin scheme

C22

4 Numerical experiments

C26

5 Conclusions

C30

References

C30

\section{Introduction}

We present an efficient algorithm to compute high order approximations to the radar cross section (RCS) of a perfectly conducting three dimensional target by solving the time harmonic Maxwell equations. The RCS of an object indicates how visible (or invisible) the object is when illuminated by an electromagnetic wave. Numerical approximations to the RCS are an essential tool for designing objects that must not be visible.

The spatial components of a time harmonic electromagnetic wave (with wavenumber $k$ ) scattered by a perfect conductor $D$ comprise an electric field $\boldsymbol{E}$ and a magnetic field $\boldsymbol{H}$ that satisfy the Maxwell equations [4, §6.1]

$$
\operatorname{curl} \boldsymbol{E}(\boldsymbol{x})-i k \boldsymbol{H}(\boldsymbol{x})=\mathbf{0}, \quad \operatorname{curl} \boldsymbol{H}(\boldsymbol{x})+i k \boldsymbol{E}(\boldsymbol{x})=\mathbf{0}, \quad \boldsymbol{x} \in \mathbb{R}^{3} \backslash \bar{D},
$$

the Silver-Müller radiation condition

$$
\lim _{|\boldsymbol{x}| \rightarrow \infty}[\boldsymbol{H}(\boldsymbol{x}) \times \boldsymbol{x}-|\boldsymbol{x}| \boldsymbol{E}(\boldsymbol{x})]=\mathbf{0},
$$


and the perfect conductor boundary condition

$$
\boldsymbol{n}(\boldsymbol{x}) \times \boldsymbol{E}(\boldsymbol{x})=-\boldsymbol{n}(\boldsymbol{x}) \times \boldsymbol{E}^{i}(\boldsymbol{x})=: \boldsymbol{f}(\boldsymbol{x}), \quad \boldsymbol{x} \in \partial D,
$$

where $\boldsymbol{n}(\boldsymbol{x})$ denotes the unit outward normal at $\boldsymbol{x} \in \partial D$. Our focus is on computing the RCS of benchmark radar targets [14] using the incident wave

$$
\boldsymbol{E}^{i}(\boldsymbol{x})=i k \hat{\boldsymbol{p}}_{0} e^{i k \boldsymbol{x} \cdot \hat{\boldsymbol{d}}_{0}}, \quad \boldsymbol{H}^{i}(\boldsymbol{x})=i k\left(\hat{\boldsymbol{d}}_{0} \times \hat{\boldsymbol{p}}_{0}\right) e^{i k \boldsymbol{x} \cdot \hat{\boldsymbol{d}}_{0}},
$$

impinging on the target $D$, where $\hat{\boldsymbol{d}}_{0}$ is the direction of the incident wave, and $\hat{\boldsymbol{p}}_{0}$ is its polarisation (perpendicular to $\hat{\boldsymbol{d}}_{0}$ ).

The RCS of the target measured by a receiver in the direction

$$
\hat{\boldsymbol{x}}=\boldsymbol{p}(\theta, \phi)=(\sin \theta \cos \phi, \sin \theta \sin \phi, \cos \theta)^{T}, \quad \theta, \phi \in \mathbb{R},
$$

is given by the scattered wave's electric far field $\boldsymbol{E}_{\infty}$, using

$$
\sigma^{\mathrm{dB}}(\hat{\boldsymbol{x}})=10 \log _{10}\left(4 \pi\left|\boldsymbol{E}_{\infty}(\hat{\boldsymbol{x}})\right|^{2} / k^{2}\right), \quad \boldsymbol{E}_{\infty}(\hat{\boldsymbol{x}})=\lim _{r \rightarrow \infty} \boldsymbol{E}(r \hat{\boldsymbol{x}}) e^{-i k r} r .
$$

Two types of RCS are of particular interest:

1. the RCS for all directions $\hat{\boldsymbol{x}}$, with a fixed incident direction $\hat{\boldsymbol{d}}_{0}$;

2. the RCS for all directions $\hat{\boldsymbol{x}}$, with varying incident directions $\hat{\boldsymbol{d}}_{0}=-\hat{\boldsymbol{x}}$.

These are respectively the bistatic and monostatic RCS [9].

The industrial standard tool for three dimensional RCS computations is the Fast Illinois Solver Code (FISC) [11]. FISC is based on a surface integral reformulation of the Maxwell equations, solved using the method of moments. To simulate medium frequency scattering using the method of moments one must solve a dense complex linear system with hundreds of thousands of unknowns. Solving such linear systems is only possible using an iterative solver, with matrix vector products computed using the fast 
multipole method. For the current state of the art in computational electromagnetics, we refer to $[1,2,5,10]$.

A spectrally accurate algorithm for electromagnetic scattering by perfect conductors utilising non-tangential harmonics was recently developed by Ganesh and Hawkins [8]. However, it is natural and convenient to use tangential functions based on vector spherical harmonics, which are widely used in many applications $[6,12,13]$. Here we describe an algorithm based on vector spherical harmonics.

Following Ganesh and Hawkins [8], we use a surface integral method for solving (1) using the unit sphere $\partial B$ as a reference surface. We assume that there exists a bijective parametrization map $\boldsymbol{q}: \partial B \rightarrow \partial D$. We demonstrate that the RCS of several objects at medium frequencies can be computed to several digits accuracy with just a few tens of thousands of unknowns. Such relatively small linear systems can be solved using a direct solver - a marked advantage in computing the monostatic RCS, which involves solving hundreds of linear systems with one matrix but hundreds of different right hand sides, arising due to co-location of transmitters and receivers.

\section{Surface integral equation}

The solution of (1)-(3) can be expressed [3, Theorem 4.19, p. 126] as a surface integral

$\boldsymbol{E}(\boldsymbol{x})=\operatorname{curl} \int_{\partial D} \Phi(\boldsymbol{x}, \boldsymbol{y}) \boldsymbol{w}(\boldsymbol{y}) d s(\boldsymbol{y}), \quad \boldsymbol{H}(\boldsymbol{x})=\frac{1}{i k} \operatorname{curl} \boldsymbol{E}(\boldsymbol{x}), \quad \boldsymbol{x} \in \mathbb{R}^{3} \backslash D$,

where $\Phi$ is the fundamental solution of the Helmholtz equation [4, Equation (2.1)] and the vector surface potential $\boldsymbol{w}$ solves the surface integral equation

$$
\boldsymbol{w}(\boldsymbol{x})+\mathfrak{M} \boldsymbol{w}(\boldsymbol{x})=2 \boldsymbol{f}(\boldsymbol{x}), \quad \boldsymbol{x} \in \partial D
$$


with

$$
\mathfrak{M} \boldsymbol{a}(\boldsymbol{x})=2 \boldsymbol{n}(\boldsymbol{x}) \times \int_{\partial D} \operatorname{curl}_{\boldsymbol{x}}\{\Phi(\boldsymbol{x}, \boldsymbol{y}) \mathcal{P}(\boldsymbol{y}) \boldsymbol{a}(\boldsymbol{y})\} d s(\boldsymbol{y}),
$$

for $\boldsymbol{a} \in C(\partial D)$ and $\mathcal{P}(\boldsymbol{y}):=I-\boldsymbol{n}(\boldsymbol{y}) \boldsymbol{n}(\boldsymbol{y})^{T}$. Here the subscript in $\operatorname{curl}_{\boldsymbol{x}}$ indicates differentiation with respect to $\boldsymbol{x}$. $\mathfrak{M}$ is weakly singular on the continuous function space $C(\partial D)$ because of the tangential projection operator $\mathcal{P}$. The solution $\boldsymbol{w}$ of (7) is tangential, because $\boldsymbol{f}$ and $\mathfrak{M} \boldsymbol{a}$ are tangential for any $\boldsymbol{a} \in C(\partial D)$. Thus (7)-(8) are equivalent to standard formulations that have $\mathcal{P}$ omitted.

Using (6) and the asymptotics of $\Phi$ [4, p. 164], the far field pattern of $\boldsymbol{E}$ is

$$
\boldsymbol{E}_{\infty}(\hat{\boldsymbol{x}})=\frac{i k}{4 \pi} \int_{\partial D} e^{-i k \hat{\boldsymbol{x}} \cdot \boldsymbol{y}} \hat{\boldsymbol{x}} \times \boldsymbol{w}(\boldsymbol{y}) d s(\boldsymbol{y}), \quad \hat{\boldsymbol{x}} \in \partial B .
$$

Thus we can compute the RCS through (9) using the solution $\boldsymbol{w}$ of (7).

The bijective parametrization $\boldsymbol{q}$ in (7) allows us to write it as

$$
\boldsymbol{W}(\hat{\boldsymbol{x}})+\mathcal{M} \boldsymbol{W}(\hat{\boldsymbol{x}})=\boldsymbol{F}(\hat{\boldsymbol{x}}), \quad \hat{\boldsymbol{x}} \in \partial B,
$$

where $\boldsymbol{F}=\boldsymbol{f} \circ \boldsymbol{q}, \boldsymbol{W}=\boldsymbol{w} \circ \boldsymbol{q}$ and $\mathcal{M}$ is an integral operator on $C(\partial B)$ derived from $\mathfrak{M}$. We refer to [8] for the full details. To implement our algorithm it is necessary to write $\mathcal{M}$ in the form

$$
\mathcal{M A}(\hat{\boldsymbol{x}})=\int_{\partial B}\left[\frac{1}{|\hat{\boldsymbol{x}}-\hat{\boldsymbol{y}}|} M_{1}(\hat{\boldsymbol{x}}, \hat{\boldsymbol{y}})+M_{2}(\hat{\boldsymbol{x}}, \hat{\boldsymbol{y}})\right] \boldsymbol{A}(\hat{\boldsymbol{y}}) d s(\hat{\boldsymbol{y}})
$$

where $M_{1}$ and $M_{2}$ are smooth, so that $\mathcal{M}=\mathcal{M}_{1}+\mathcal{M}_{2}$, with $\mathcal{M}_{1}$ and $\mathcal{M}_{2}$ having respectively weakly singular and smooth kernels. In this article we use $\mathcal{M}_{1}$ and $\mathcal{M}_{2}$ as previously given by Ganesh and Hawkins [8, Equations (2.11)-(2.12)]. 


\section{High order fully discrete Galerkin scheme}

In this section we describe our high-order fully discrete Galerkin scheme for (10). We seek an approximation to the solution of (10) in the space

$$
\mathbb{Q}_{n}=\operatorname{span}\left\{\boldsymbol{U}_{l, j}, \boldsymbol{V}_{l, j}, \boldsymbol{W}_{l, j}: 0 \leq l \leq n,|j| \leq l\right\},
$$

where

$$
\boldsymbol{U}_{l, j}(\hat{\boldsymbol{x}})=\frac{1}{\sqrt{l(l+1)}} \operatorname{Grad} Y_{l, j}(\hat{\boldsymbol{x}}), \quad \boldsymbol{V}_{l, j}(\hat{\boldsymbol{x}})=\hat{\boldsymbol{x}} \times \boldsymbol{U}_{l, j}(\hat{\boldsymbol{x}}), \quad \hat{\boldsymbol{x}} \in \partial B,
$$

for $1 \leq l \leq n$ and $|j| \leq l$, with $\boldsymbol{U}_{0,0}=\boldsymbol{V}_{0,0}=\mathbf{0}$, and

$$
\boldsymbol{W}_{l, j}(\hat{\boldsymbol{x}})=\hat{\boldsymbol{x}} Y_{l, j}(\hat{\boldsymbol{x}}), \quad 0 \leq l \leq n, \quad|j| \leq l, \quad \hat{\boldsymbol{x}} \in \partial B,
$$

where Grad is the surface gradient on $\partial B$ [4, p. 167], and $Y_{l, j}$ is a normalised spherical harmonic [8, Equation (3.2)]. It is convenient to write

$$
\left[\boldsymbol{U}_{l, j}, \boldsymbol{V}_{l, j}, \boldsymbol{W}_{l, j}\right](\hat{\boldsymbol{x}})=\left[\boldsymbol{v}_{1}, \boldsymbol{v}_{2}, \boldsymbol{v}_{3}\right](\theta, \phi) \Omega_{l, j}(\theta) e^{i j \phi},
$$

where vectors $\boldsymbol{v}_{1}, \boldsymbol{v}_{2}$ and $\boldsymbol{v}_{3}$ depend only on $\theta, \phi$ and $\Omega_{l, j}(\theta)$ is a $3 \times 3$ matrix.

The inner product integrals in the Galerkin scheme are approximated numerically using the $2(m+1) \times(m+1)$ rectangle Gauss rule

$$
\int_{\partial B} G(\hat{\boldsymbol{x}}) d s(\hat{\boldsymbol{x}}) \approx Q_{m}(G)=\sum_{r=0}^{2 m+1} \sum_{s=1}^{m+1} \mu_{r}^{m} \nu_{s}^{m} G\left(\hat{\boldsymbol{z}}_{r s}^{m}\right), \quad G \in C(\partial B),
$$

where $\hat{\boldsymbol{z}}_{r s}^{m}=\boldsymbol{p}\left(\theta_{s}^{m}, \phi_{r}^{m}\right), \theta_{s}^{m}$ and $\nu_{s}^{m}$ are the Gauss-Legendre points and weights respectively and $\phi_{r}^{m}$ and $\mu_{r}^{m}$ are the rectangle rule points and weights respectively. The rectangle Gauss rule is exact for spherical polynomials of degree $2 m$. Let

$$
(\boldsymbol{G}, \boldsymbol{H})_{m}:=Q_{m}\left(\overline{\boldsymbol{H}}^{T} \boldsymbol{G}\right), \quad \boldsymbol{G}, \boldsymbol{H} \in C(\partial B) .
$$


Since each element in $\mathbb{Q}_{n}$ is a polynomial of degree at most $n+1,(\cdot, \cdot)_{n+1}$ is a discrete inner product on the $N\left(=3(n+1)^{2}-2\right)$ dimensional space $\mathbb{Q}_{n}$.

Using (13), our Galerkin approximation to the solution of (10) in $\mathbb{Q}_{n}$ is

$$
\Psi_{n}=\sum_{l=0}^{n} \sum_{|j| \leq l}\left[\boldsymbol{U}_{l, j}, \boldsymbol{V}_{l, j}, \boldsymbol{W}_{l, j}\right] \mathrm{w}_{l j},
$$

where each $\mathrm{w}_{l j}$ is a 3 -vector and $\mathrm{w}=\left(\mathrm{w}_{l j}\right)$ solves $(\mathrm{I}+\mathrm{M}) \mathrm{w}=\mathrm{f}$ with (in block form)

$$
\begin{aligned}
M_{l^{\prime} j^{\prime}, l j} & =\left(\mathcal{M}_{n^{\prime}}\left[\boldsymbol{U}_{l, j}, \boldsymbol{V}_{l, j}, \boldsymbol{W}_{l, j}\right],\left[\boldsymbol{U}_{l^{\prime}, j^{\prime}}, \boldsymbol{V}_{l^{\prime}, j^{\prime}}, \boldsymbol{W}_{l^{\prime}, j^{\prime}}\right]\right)_{n+1}, \\
\mathrm{f}_{l^{\prime} j^{\prime}} & =\left(\boldsymbol{f},\left[\boldsymbol{U}_{l^{\prime}, j^{\prime}}, \boldsymbol{V}_{l^{\prime}, j^{\prime}}, \boldsymbol{W}_{l^{\prime}, j^{\prime}}\right]\right)_{n+1}
\end{aligned}
$$

where $\mathcal{M}_{n^{\prime}}$ is a high-order approximation to $\mathcal{M}$ described below, with $n^{\prime}=$ $\alpha n$ for $\alpha>1$. Here each $\mathrm{f}_{l^{\prime}, j^{\prime}}$ is a 3 -vector and each $\mathrm{M}_{l^{\prime} j^{\prime}, l j}$ is a $3 \times 3$ matrix.

Following Ganesh and Hawkins [8], we approximate the kernel of $\mathcal{M}$ in a rotated coordinate system on $\partial B$, under which the weak singularity in $\mathcal{M}_{1}$ appears at the north pole. To this end, for each $\hat{\boldsymbol{x}} \in \partial B$, we denote by $T_{\hat{\boldsymbol{x}}}$ the orthogonal matrix that maps $\hat{\boldsymbol{x}}$ to the north pole $\hat{\boldsymbol{n}}=(0,0,1)^{T}$. The matrix $T_{\hat{\boldsymbol{x}}}$ induces a linear transformation and its bivariate analogue,

$$
\begin{aligned}
& \mathcal{T}_{\hat{\boldsymbol{x}}} \boldsymbol{A}(\hat{\boldsymbol{z}}):=\boldsymbol{A}\left(T_{\hat{\boldsymbol{x}}}^{-1} \hat{\boldsymbol{z}}\right), \quad \hat{\boldsymbol{z}} \in \partial B, \quad \boldsymbol{A} \in C(\partial B), \\
& \mathcal{T}_{\hat{\boldsymbol{x}}} \boldsymbol{A}\left(\hat{\boldsymbol{z}}_{1}, \hat{\boldsymbol{z}}_{2}\right):=\boldsymbol{A}\left(T_{\hat{\boldsymbol{x}}}^{-1} \hat{\boldsymbol{z}}_{1}, T_{\hat{\boldsymbol{x}}}^{-1} \hat{\boldsymbol{z}}_{2}\right), \quad \hat{\boldsymbol{z}}_{i} \in \partial B, \quad \boldsymbol{A} \in C(\partial B \times \partial B) .
\end{aligned}
$$

Using the orthogonality of $T_{\hat{\boldsymbol{x}}}$ we have $|\hat{\boldsymbol{x}}-\hat{\boldsymbol{y}}|=\left|T_{\hat{\boldsymbol{x}}}^{-1}(\hat{\boldsymbol{n}}-\hat{\boldsymbol{z}})\right|=|\hat{\boldsymbol{n}}-\hat{\boldsymbol{z}}|$ for $\hat{\boldsymbol{x}}, \hat{\boldsymbol{z}} \in \partial B$ and $\hat{\boldsymbol{y}}=T_{\hat{\boldsymbol{x}}}^{-1} \hat{\boldsymbol{z}}$. Since the surface measure on $\partial B$ is invariant under an orthogonal transformation,

$$
\mathcal{M}_{1} \boldsymbol{A}(\hat{\boldsymbol{x}})=\int_{\partial B} \frac{1}{|\hat{\boldsymbol{n}}-\hat{\boldsymbol{z}}|} \mathcal{T}_{\hat{\boldsymbol{x}}} M_{1}(\hat{\boldsymbol{n}}, \hat{\boldsymbol{z}}) \mathcal{T}_{\hat{\boldsymbol{x}}} \boldsymbol{A}(\hat{\boldsymbol{z}}) d s(\hat{\boldsymbol{z}}), \quad \boldsymbol{A} \in C(\partial B) .
$$


Using the discrete inner product (13), we define the fully discrete orthogonal projection operator $\mathcal{O}_{n}: C(\partial B) \rightarrow \mathbb{Q}_{n}$,

$$
\mathcal{O}_{n} \boldsymbol{A}=\sum_{l=0}^{n} \sum_{|j| \leq l}\left[\boldsymbol{U}_{l, j}, \boldsymbol{V}_{l, j}, \boldsymbol{W}_{l, j}\right]\left(\boldsymbol{A},\left[\boldsymbol{U}_{l, j}, \boldsymbol{V}_{l, j}, \boldsymbol{W}_{l, j}\right]\right)_{n+1}, \quad \boldsymbol{A} \in C(\partial B) .
$$

Let $\boldsymbol{A} \in C(\partial B)$ and $\hat{\boldsymbol{x}} \in \partial B$. Using (19), we approximate $\mathcal{M}_{1} \boldsymbol{A}(\hat{\boldsymbol{x}})$ by

$$
\begin{aligned}
\mathcal{M}_{1, n^{\prime}} \boldsymbol{A}(\hat{\boldsymbol{x}})= & \int_{\partial B} \frac{1}{|\hat{\boldsymbol{n}}-\hat{\boldsymbol{z}}|} \mathcal{O}_{n^{\prime}}\left\{\mathcal{T}_{\hat{\boldsymbol{x}}} M_{1}(\hat{\boldsymbol{n}}, \cdot) \mathcal{T}_{\hat{\boldsymbol{x}}} \boldsymbol{A}(\cdot)\right\}(\hat{\boldsymbol{z}}) d s(\hat{\boldsymbol{z}}) \\
= & \sum_{l=0}^{n^{\prime}} \sum_{|j| \leq l}\left(\int_{\partial B} \frac{1}{|\hat{\boldsymbol{n}}-\hat{\boldsymbol{z}}|}\left[\boldsymbol{U}_{l, j}, \boldsymbol{V}_{l, j}, \boldsymbol{W}_{l, j}\right](\hat{\boldsymbol{z}}) d s(\hat{\boldsymbol{z}})\right) \\
& \cdot\left(\mathcal{T}_{\hat{\boldsymbol{x}}} M_{1}(\hat{\boldsymbol{n}}, \cdot) \mathcal{T}_{\hat{\boldsymbol{x}}} \boldsymbol{A}(\cdot),\left[\boldsymbol{U}_{l, j}, \boldsymbol{V}_{l, j}, \boldsymbol{W}_{l, j}\right](\cdot)\right)_{n^{\prime}+1} .
\end{aligned}
$$

Using [6, Theorem 12.7.6, p. 343], we have for any $\hat{\boldsymbol{x}} \in \partial B$,

$$
\int_{\partial B} \frac{1}{|\hat{\boldsymbol{x}}-\hat{\boldsymbol{z}}|}\left[\boldsymbol{U}_{l, j}, \boldsymbol{V}_{l, j}, \boldsymbol{W}_{l, j}\right](\hat{\boldsymbol{z}}) d s(\hat{\boldsymbol{z}})=\left[\boldsymbol{U}_{l, j}, \boldsymbol{V}_{l, j}, \boldsymbol{W}_{l, j}\right](\hat{\boldsymbol{x}}) \Lambda_{l}
$$

where $\Lambda_{l}$ is a $3 \times 3$ matrix that depends only on $l$. Using (20) and expanding the discrete inner product using (13), our approximation simplifies to

$$
\begin{aligned}
& \mathcal{M}_{1, n^{\prime}} \boldsymbol{A}(\hat{\boldsymbol{x}})=\sum_{r^{\prime}=0}^{2 n^{\prime}+3} \sum_{s^{\prime}=1}^{n^{\prime}+2} \mu_{r^{\prime}}^{n^{\prime}+1} \nu_{s^{\prime}}^{n^{\prime}+1} \sum_{l=0}^{n^{\prime}} \sum_{|j| \leq l}\left[\boldsymbol{U}_{l, j}, \boldsymbol{V}_{l, j}, \boldsymbol{W}_{l, j}\right](\hat{\boldsymbol{n}}) \Lambda_{l} \\
& \overline{\left[\boldsymbol{U}_{l, j}, \boldsymbol{V}_{l, j}, \boldsymbol{W}_{l, j}\right]\left(\hat{\boldsymbol{z}}_{r^{\prime} s^{\prime}}^{n^{\prime}+1}\right)^{T}} \mathcal{T}_{\hat{\boldsymbol{x}}} M_{1}\left(\hat{\boldsymbol{n}}, \hat{\boldsymbol{z}}_{r^{\prime} s^{\prime}}^{n^{\prime}+1}\right) \mathcal{T}_{\hat{\boldsymbol{x}}} \boldsymbol{A}\left(\hat{\boldsymbol{z}}_{r^{\prime} s^{\prime}}^{n^{\prime}+1}\right) .
\end{aligned}
$$

The vector spherical harmonics satisfy an addition rule [6, Theorem 12.6.1, p. 335], from which we have

$$
\sum_{|j| \leq l}\left[\boldsymbol{U}_{l, j}, \boldsymbol{V}_{l, j}, \boldsymbol{W}_{l, j}\right](\hat{\boldsymbol{x}}) \Lambda_{l}{\overline{\left[\boldsymbol{U}_{l, j}, \boldsymbol{V}_{l, j}, \boldsymbol{W}_{l, j}\right](\hat{\boldsymbol{y}}}}^{T}=\Pi_{l}(\hat{\boldsymbol{x}}, \hat{\boldsymbol{y}}), \quad \hat{\boldsymbol{x}}, \hat{\boldsymbol{y}} \in \partial B
$$


where $\Pi_{l}(\hat{\boldsymbol{x}}, \hat{\boldsymbol{y}})$ can be written in terms of $\hat{\boldsymbol{x}}$ and $\hat{\boldsymbol{y}}$ and Legendre polynomials of degree $l$ (and their first two derivatives) evaluated at $\hat{\boldsymbol{x}} \cdot \hat{\boldsymbol{y}}$. Using the addition rule and defining $\Upsilon_{r^{\prime} s^{\prime}}^{n^{\prime}+1}=\sum_{l=0}^{n^{\prime}} \Pi_{l}\left(\hat{\boldsymbol{n}}, \hat{\boldsymbol{z}}_{r^{\prime} s^{\prime}}^{n^{\prime}+1}\right)$, our approximation simplifies to

$$
\mathcal{M}_{1, n^{\prime}} \boldsymbol{A}(\hat{\boldsymbol{x}})=\sum_{r^{\prime}=0}^{2 n^{\prime}+3} \sum_{s^{\prime}=1}^{n^{\prime}+2} \mu_{r^{\prime}}^{n^{\prime}+1} \nu_{s^{\prime}}^{n^{\prime}+1} \Upsilon_{r^{\prime} s^{\prime}}^{n^{\prime}+1} \mathcal{T}_{\hat{\boldsymbol{x}}} M_{1}\left(\hat{\boldsymbol{n}}, \hat{\boldsymbol{z}}_{r^{\prime} s^{\prime}}^{n^{\prime}+1}\right) \mathcal{T}_{\hat{\boldsymbol{x}}} \boldsymbol{A}\left(\hat{\boldsymbol{z}}_{r^{\prime} s^{\prime}}^{n^{\prime}+1}\right)
$$

It is convenient to use a similar approximation for both $\mathcal{M}_{1}$ and $\mathcal{M}_{2}$.

$$
\begin{aligned}
\mathcal{M}_{2, n^{\prime}} \boldsymbol{A}(\hat{\boldsymbol{x}})= & \int_{\partial B} \mathcal{O}_{n^{\prime}}\left\{\mathcal{T}_{\hat{\boldsymbol{x}}} M_{2}(\hat{\boldsymbol{n}}, \cdot) \mathcal{T}_{\hat{\boldsymbol{x}}} \boldsymbol{A}(\cdot)\right\}(\hat{\boldsymbol{z}}) d s(\hat{\boldsymbol{z}}) \\
= & \sum_{l=0}^{n^{\prime}} \sum_{|j| \leq l}\left(\int_{\partial B}\left[\boldsymbol{U}_{l, j}, \boldsymbol{V}_{l, j}, \boldsymbol{W}_{l, j}\right](\hat{\boldsymbol{z}}) d s(\hat{\boldsymbol{z}})\right) \\
& \cdot\left(\mathcal{T}_{\hat{\boldsymbol{x}}} M_{2}(\hat{\boldsymbol{n}}, \cdot) \mathcal{T}_{\hat{\boldsymbol{x}}} \boldsymbol{A}(\cdot),\left[\boldsymbol{U}_{l, j}, \boldsymbol{V}_{l, j}, \boldsymbol{W}_{l, j}\right](\cdot)\right)_{n^{\prime}+1} .
\end{aligned}
$$

The integrals of $\boldsymbol{U}_{l, j}, \boldsymbol{V}_{l, j}$ and $\boldsymbol{W}_{l, j}$ over $\partial B$ are zero for $l>1$ and the nonzero cases are easily evaluated analytically. For convenience, we write

$$
\int_{\partial B}\left[\boldsymbol{U}_{l, j}, \boldsymbol{V}_{l, j}, \boldsymbol{W}_{l, j}\right](\hat{\boldsymbol{z}}) d s(\hat{\boldsymbol{z}})=\Delta_{l, j}
$$

Defining $\widetilde{\Upsilon}_{r^{\prime} s^{\prime}}^{n^{\prime}+1}=\sum_{l=0}^{1} \sum_{|j| \leq l} \Delta_{l, j}{\overline{\left.\boldsymbol{U}_{l, j}, \boldsymbol{V}_{l, j}, \boldsymbol{W}_{l, j}\right]\left(\hat{\boldsymbol{z}}_{r^{\prime} s^{\prime}}^{n^{\prime}+1}\right)}}^{T}$ and expanding the discrete inner product using (13), we have

$$
\mathcal{M}_{2, n^{\prime}} \boldsymbol{A}(\hat{\boldsymbol{x}})=\sum_{r^{\prime}=0}^{2 n^{\prime}+3} \sum_{s^{\prime}=1}^{n^{\prime}+2} \mu_{r^{\prime}}^{n^{\prime}+1} \nu_{s^{\prime}}^{n^{\prime}+1} \widetilde{\Upsilon}_{r^{\prime} s^{\prime}}^{n^{\prime}+1} \mathcal{T}_{\hat{\boldsymbol{x}}} M_{2}\left(\hat{\boldsymbol{n}}, \hat{\boldsymbol{z}}_{r^{\prime} s^{\prime}}^{n^{\prime}+1}\right) \mathcal{T}_{\hat{\boldsymbol{x}}} \boldsymbol{A}\left(\hat{\boldsymbol{z}}_{r^{\prime} s^{\prime}}^{n^{\prime}+1}\right)
$$

Using (21) and (22), our high-order approximation to $\mathcal{M}$ is $\mathcal{M}_{n^{\prime}}=$ $\mathcal{M}_{1, n^{\prime}}+\mathcal{M}_{2, n^{\prime}}$. To ensure high order approximation, we choose $n^{\prime}=2 n$ in all of the numerical experiments described in Section 4. 

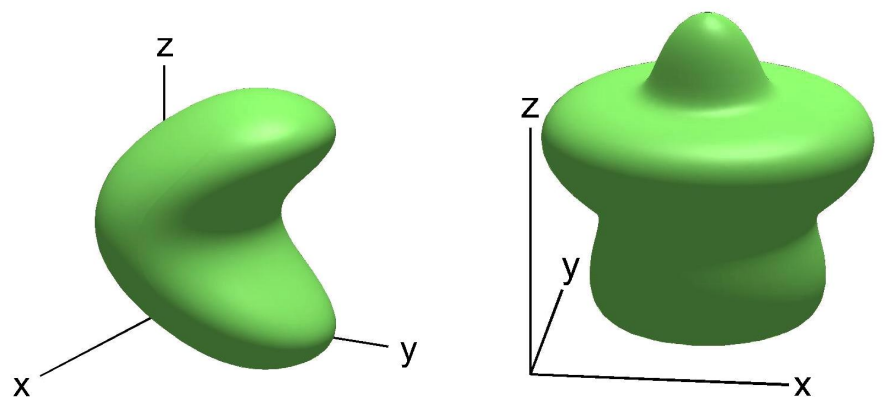

FiguRE 1: Bean and fountain shaped obstacles.

Using [6, Equation (12.7.4), p. 341], each rotated vector spherical harmonic can be written as a linear combination of vector spherical harmonics of the same degree. A stable and efficient representation is [7, p. 224]

$$
\mathcal{T}_{\hat{\boldsymbol{x}}_{r s}}\left[\boldsymbol{U}_{l, j}, \boldsymbol{V}_{l, j}, \boldsymbol{W}_{l, j}\right]=T_{\hat{\boldsymbol{x}}_{r s}}^{-1} \sum_{|\tilde{j}| \leq l} F_{s l \tilde{j} j} e^{i(j-\tilde{j}) \phi_{r}^{n+1}}\left[\boldsymbol{U}_{l, j}, \boldsymbol{V}_{l, j}, \boldsymbol{W}_{l, j}\right],
$$

for previously recorded coefficients $F_{s l \tilde{j} j}$ [7, p. 224]. Using (11) and (23), the $N \times N$ matrix in (15) can be efficiently assembled in $O\left(N^{2.5}\right)$ operations [8, $\S 3.3]$.

\section{$4 \quad$ Numerical experiments}

We demonstrate the efficiency of our algorithm with RCS computations for several smooth and non-smooth perfectly conducting obstacles. The bean and fountain shaped smooth obstacles are shown in Figure 1. The nonsmooth obstacles are benchmark radar targets NASA almond, cone-sphere (see Figure 2) and ogive [14]. The number of unknowns required to solve each problem depends on the shape of the obstacle and its electromagnetic size, 
TABle 1: Performance of FISC [11, p. 28] and GH (present). Scattering of a plane wave by a sphere of diameter $24 \lambda$.

\begin{tabular}{ccccc} 
Algorithm & Unknowns & Computer/CPU & CPU time & RMS error \\
\hline FISC & 602112 & SGI R8000 & $12 \mathrm{~h}$ & $3.0 \mathrm{e}-01$ \\
GH & $13120(n=80)$ & $4 \times \mathrm{DcOp}$ & $0.47 \mathrm{~h}$ & $5.7 \mathrm{e}-02$ \\
GH & $15840(n=88)$ & $4 \times \mathrm{DcOp}$ & $0.74 \mathrm{~h}$ & $1.5 \mathrm{e}-05$ \\
\hline
\end{tabular}

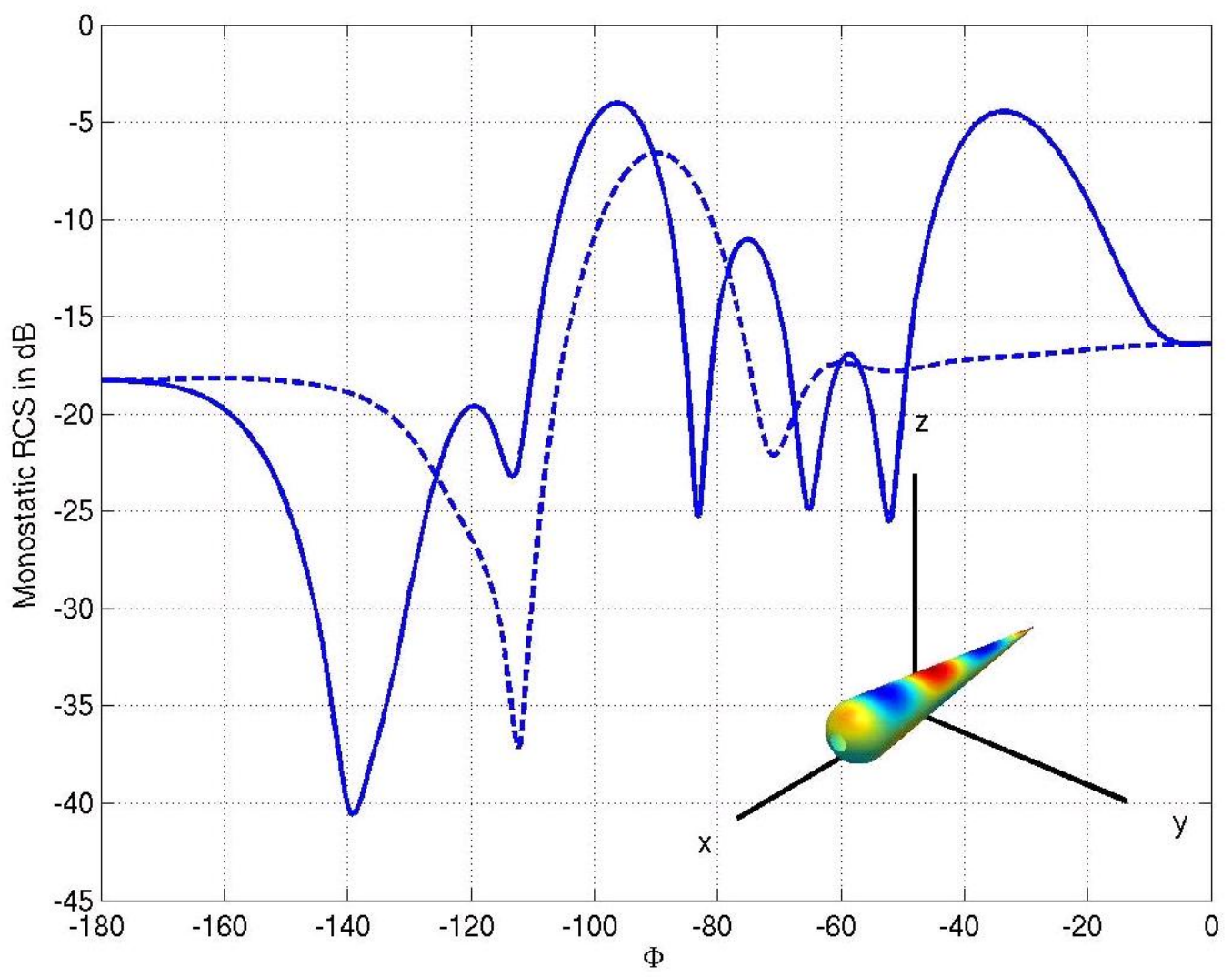

FiguRE 2: Monostatic RCS of the cone-sphere with electromagnetic size $2 \lambda$ : Numerical using GH. 


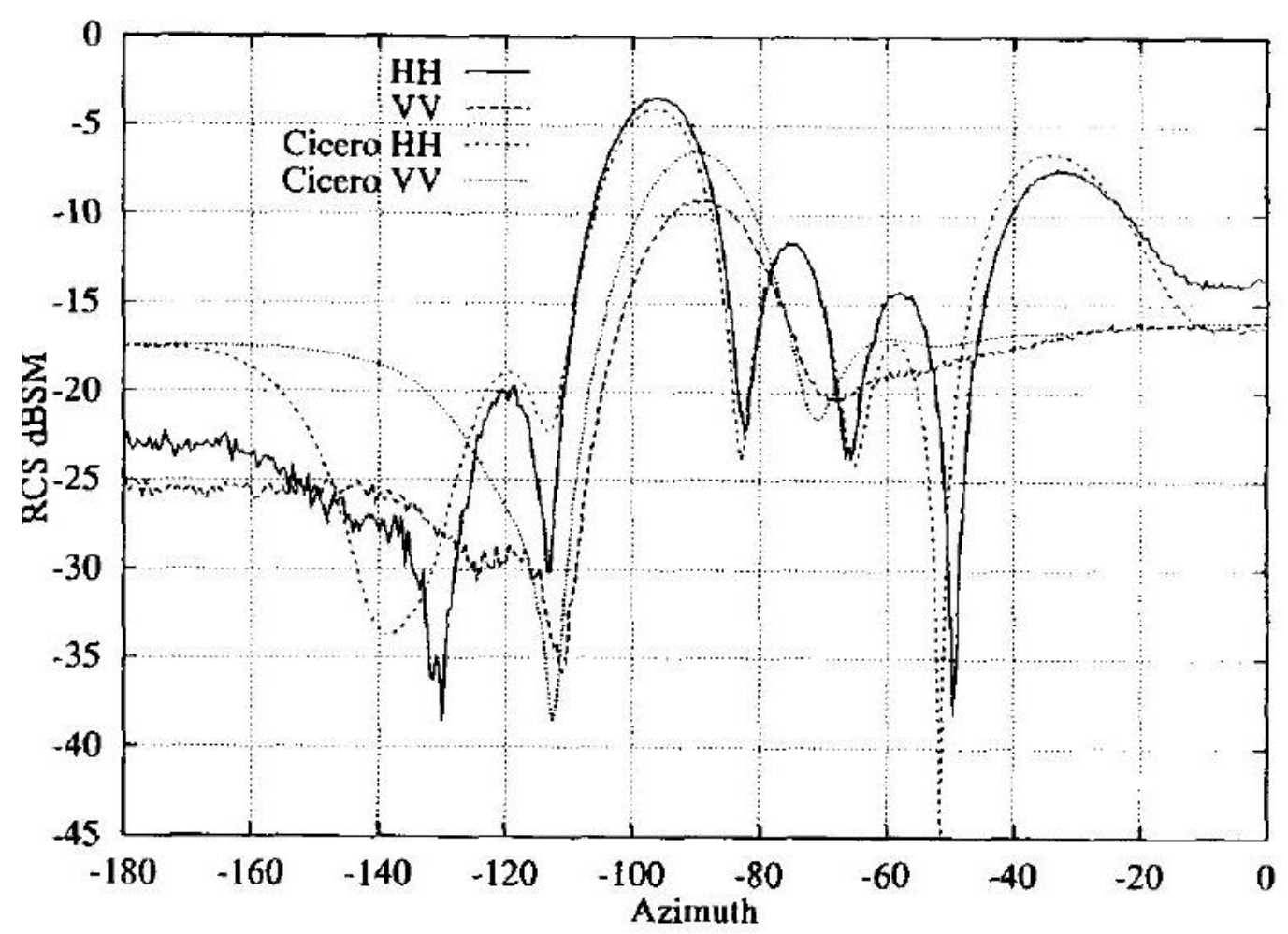

FigurE 3: Monostatic RCS of the cone-sphere with electromagnetic size $2 \lambda$ : Experimental and Cicero [14]. 
TABLE 2: Convergence of backscattered RCS.

\begin{tabular}{ccccc} 
& bean & fount & almond & ogive \\
& size $16 \lambda$ & size $16 \lambda$ & size $\lambda$ & size $\lambda$ \\
& $n_{0}=85$ & $n_{0}=95$ & $n_{0}=75$ & $n_{0}=35$ \\
$n-n_{0}$ & $\sigma_{n}^{\mathrm{dB}}\left(\pi / 2,89.9^{\circ}\right)$ & $\sigma_{n}^{\mathrm{dB}}\left(\pi / 2,0.0^{\circ}\right)$ & $\sigma_{n}^{\mathrm{dB}}\left(\pi / 2,0.0^{\circ}\right)$ & $\sigma_{n}^{\mathrm{dB}}\left(\pi / 2,0.0^{\circ}\right)$ \\
\hline 0 & 14.33379 & -2.20856 & -27.31434 & -42.60732 \\
10 & 14.33375 & -2.20858 & -27.28491 & -42.61177 \\
20 & 14.33375 & -2.20858 & -27.26151 & -42.61367
\end{tabular}

TABLE 3: Convergence of monostatic RCS for cone-sphere with electromagnetic size $2 \lambda$.

\begin{tabular}{ccccc}
$n$ & $\sigma_{n}^{\mathrm{dB}}\left(\pi / 2,0.0^{\circ}\right)$ & $\sigma_{n}^{\mathrm{dB}}\left(\pi / 2,59.9^{\circ}\right)$ & $\sigma_{n}^{\mathrm{dB}}\left(\pi / 2,120.1^{\circ}\right)$ & $\sigma_{n}^{\mathrm{dB}}\left(\pi / 2,180.0^{\circ}\right)$ \\
\hline 85 & -16.39436 & -17.17169 & -19.71477 & -18.33426 \\
95 & -16.36141 & -17.20299 & -19.60757 & -18.23749 \\
105 & -16.34336 & -17.22089 & -19.52198 & -18.18573 \\
\hline
\end{tabular}

computed as the diameter of the obstacle divided by the incident wavelength $\lambda=2 \pi / k$.

We obtained all results in this section using a parallel implementation of our algorithm on a multiprocessor computing cluster with $2 \mathrm{GHz}$ dual core, Opteron (DcOp) processors. In Table 1, for the case of scattering by a sphere of electromagnetic size $24 \lambda$, we compare our simulated RCS with the exact RCS (given by the Mie series), and the CPU time with that of FISC [11]. Very good accuracy and substantial reduction in computing time are achieved using our algorithm with only 2.5 percent of the unknowns required by FISC.

The relatively small number of unknowns required by our algorithm is further demonstrated in Tables $2-3$ by tabulating values of the RCS at a number of directions. (We denote the RCS at $\boldsymbol{p}(\theta, \phi)$ by $\sigma^{\mathrm{dB}}(\theta, \phi)$.) In the case of the smooth obstacles, only a few tens of thousands of unknowns are required to compute the RCS to several digits accuracy. Only a few digits 
of global accuracy is expected for scattering by non-smooth obstacles. The tabulated results demonstrate that only a few tens of thousands of unknowns are required to obtain visually accurate RCS of non-smooth obstacles.

For the cone-sphere example, we also demonstrate the accuracy of our simulated RCS values (labelled GH) in Figures 2-3 by comparison with experimental and numerical RCS values in [14].

\section{Conclusions}

The algorithm presented above has been shown to give several digits accuracy in the computed RCS in just a few hours. The advantage of this algorithm over current industrial standard algorithms is that it can compute the monostatic and bistatic RCS in about the same amount of time.

\section{References}

[1] A. Bendali, Y. Boubendir, and M. Fares. A FETI-like domain decomposition method for coupling finite elements and boundary elements in large-size scattering problems of acoustic scattering. Computers and Structures, 85:526-535, 2007. doi:10.1016/j.compstruc.2006.08.029. C20

[2] W. C. Chew, J. M. Jin, E. Michielssen, and J. Song. Fast and Efficient Algorithms in Computational Electromagnetics. Artech House, 2001. $\mathrm{C} 20$

[3] D. Colton and R. Kress. Integral Equation Methods in Scattering Theory. Wiley, 1983. C20 
[4] D. Colton and R. Kress. Inverse Acoustic and Electromagnetic Scattering Theory. Springer, 1998. C18, C20, C21, C22

[5] E. Darve and P. Havé. Efficient fast multipole method for low-frequency scattering. J. Comput. Phys., 197:341-363, 2004. doi:10.1016/j.jcp.2003.12.002. C20

[6] W. Freeden, T. Gervens, and M. Schreiner. Constructive Approximation on the Sphere. Oxford University Press, 1998. C20, C24, C26

[7] M. Ganesh and I. G. Graham. A high-order algorithm for obstacle scattering in three dimensions. J. Comput. Phys., 198:211-242, 2004. doi:10.1016/j.jcp.2004.01.007. C26

[8] M. Ganesh and S. C. Hawkins. A spectrally accurate algorithm for electromagnetic scattering in three dimensions. Numer. Algorithms, 43:25-60, 2006. doi:10.1007/s11075-006-9033-7. C20, C21, C22, C23, $\mathrm{C} 26$

[9] E. F. Knott, J. F. Shaeffer, and M. T. Tuley. Radar Cross Section. SciTech Publishing Inc, 2004. C19

[10] P. Monk. Finite Element Methods for Maxwell's Equations. Oxford University Press, 2003. C20

[11] J. M. Song, C. C. Lu, W. C. Chew, and S. W. Lee. Fast Illinois Solver Code (FISC). IEEE Antennas Propag. Mag., 40(3):27-34, 1998. doi:10.1109/74.706067. C19, C27, C29

[12] R. A. R. Tricker. Introduction to Meteorological Optics. Elsevier, 1970. $\mathrm{C} 20$

[13] R. T. Wang and H. C. van de Hulst. Rainbows: Mie computations and the Airy approximation. Appl. Optics, 30:106-117, 1991.

http://www.opticsinfobase.org/abstract.cfm?URI=ao-30-1-106. $\mathrm{C} 20$ 
[14] A. C. Woo, H. T. Wang, M. J. Schuh, and M. L. Sanders. Benchmark radar targets for the validation of computational electromagnetics programs. IEEE Antennas Propag. Mag., 35(1):84-89, 1993. doi:10.1109/74.210840. C19, C26, C28, C30 


\section{Author addresses}

1. M. Ganesh, Department of Mathematical and Computer Sciences, Colorado School of Mines, Golden, CO 80401, USA. mailto:mganesh@mines. edu

2. S. C. Hawkins, School of Mathematics and Statistics, University of New South Wales, Sydney, NSW 2052, Australia.

mailto: stuart@maths. unsw.edu . au 